\title{
Capturing Provenance of Data Curation at BCO-DMO (n)
}

Adam Shepherd Amber York

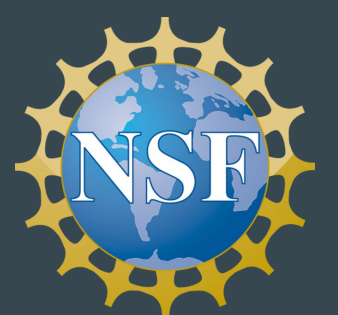




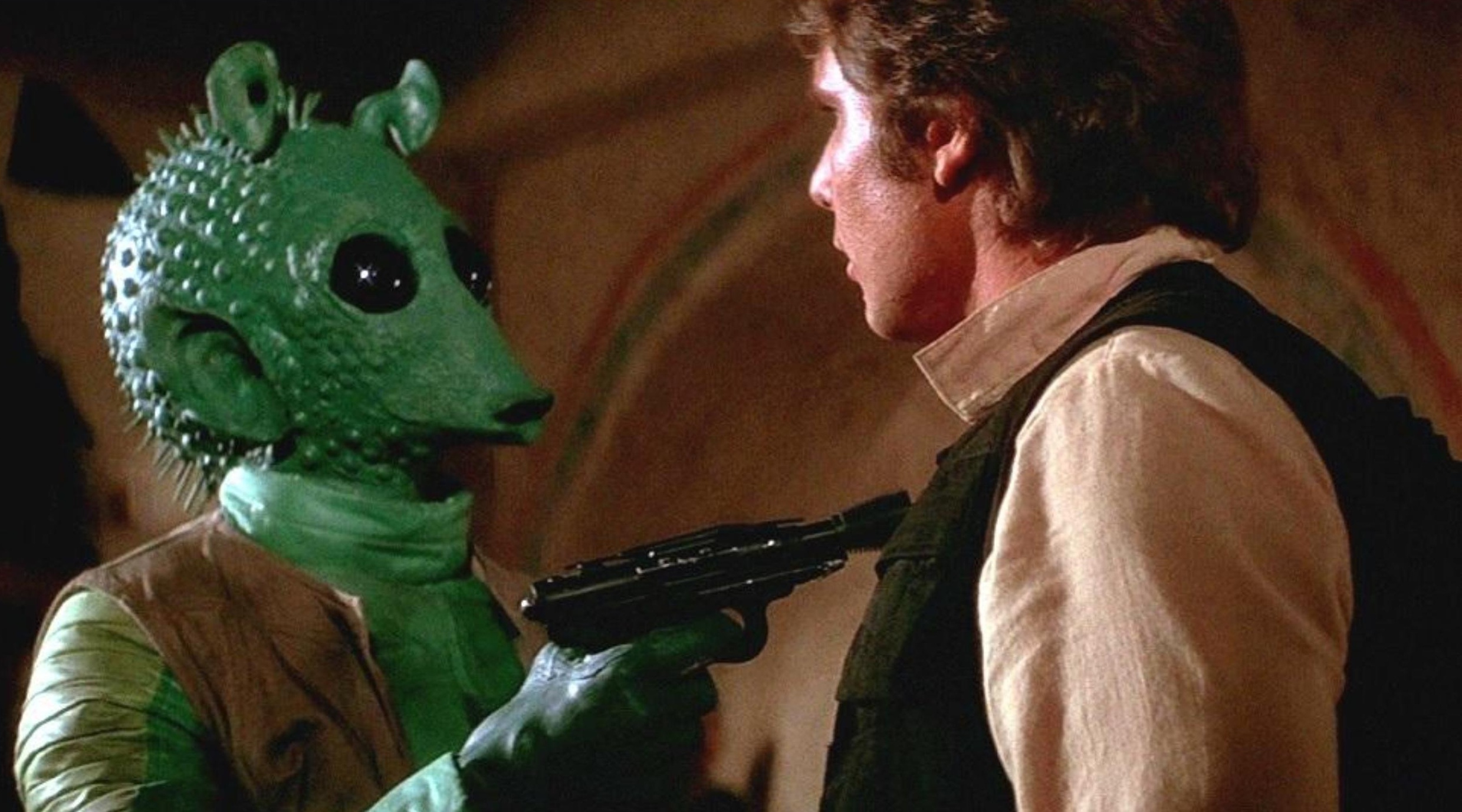



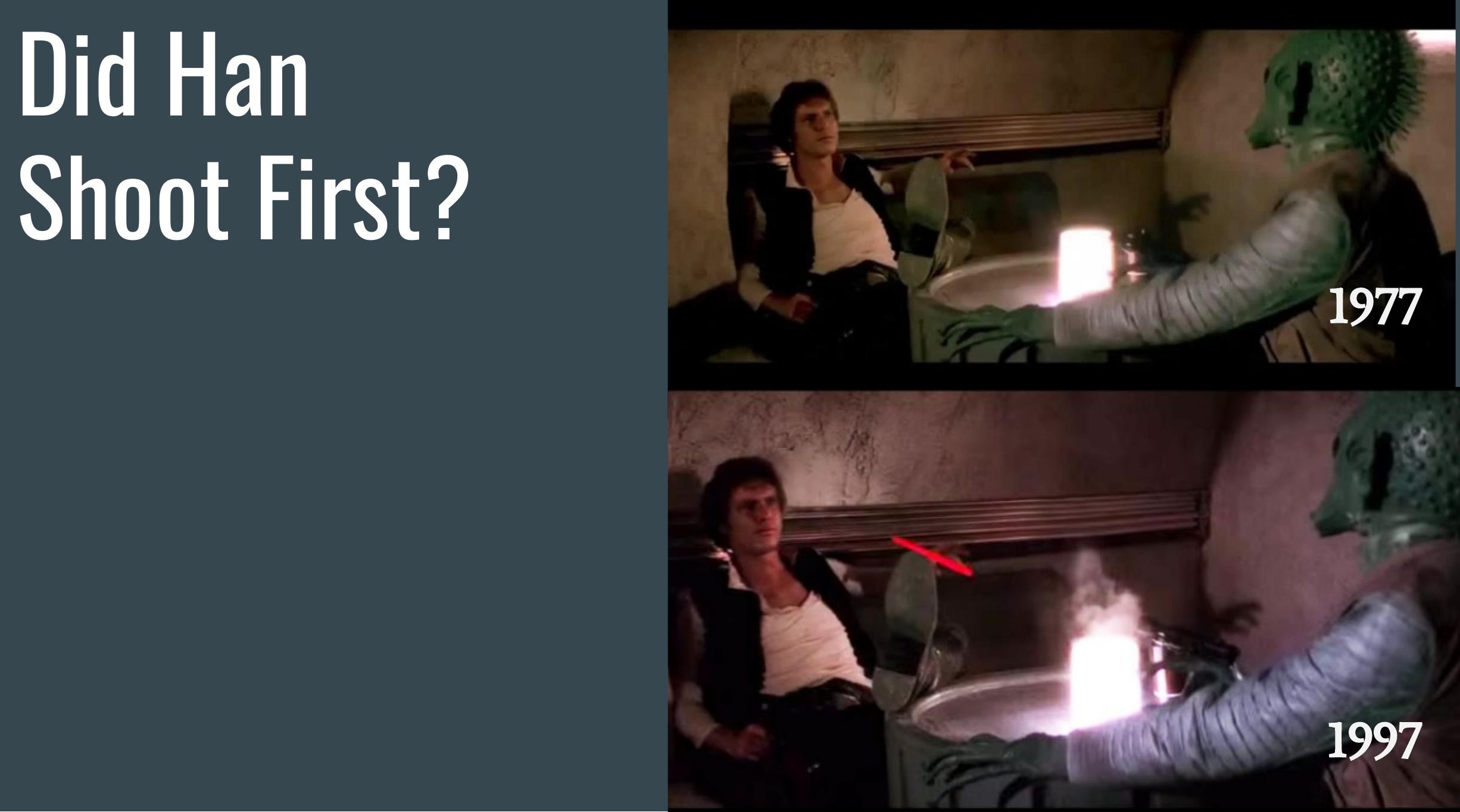


\section{From the Author:}

"To me, [the original movie] doesn't really exist anymore. ...

I'm sorry you saw half a completed film and fell in love with it.

But I want it to be the way I want it to be."

- George Lucas, 2004 interview 
1997: A Disturbance in the Force
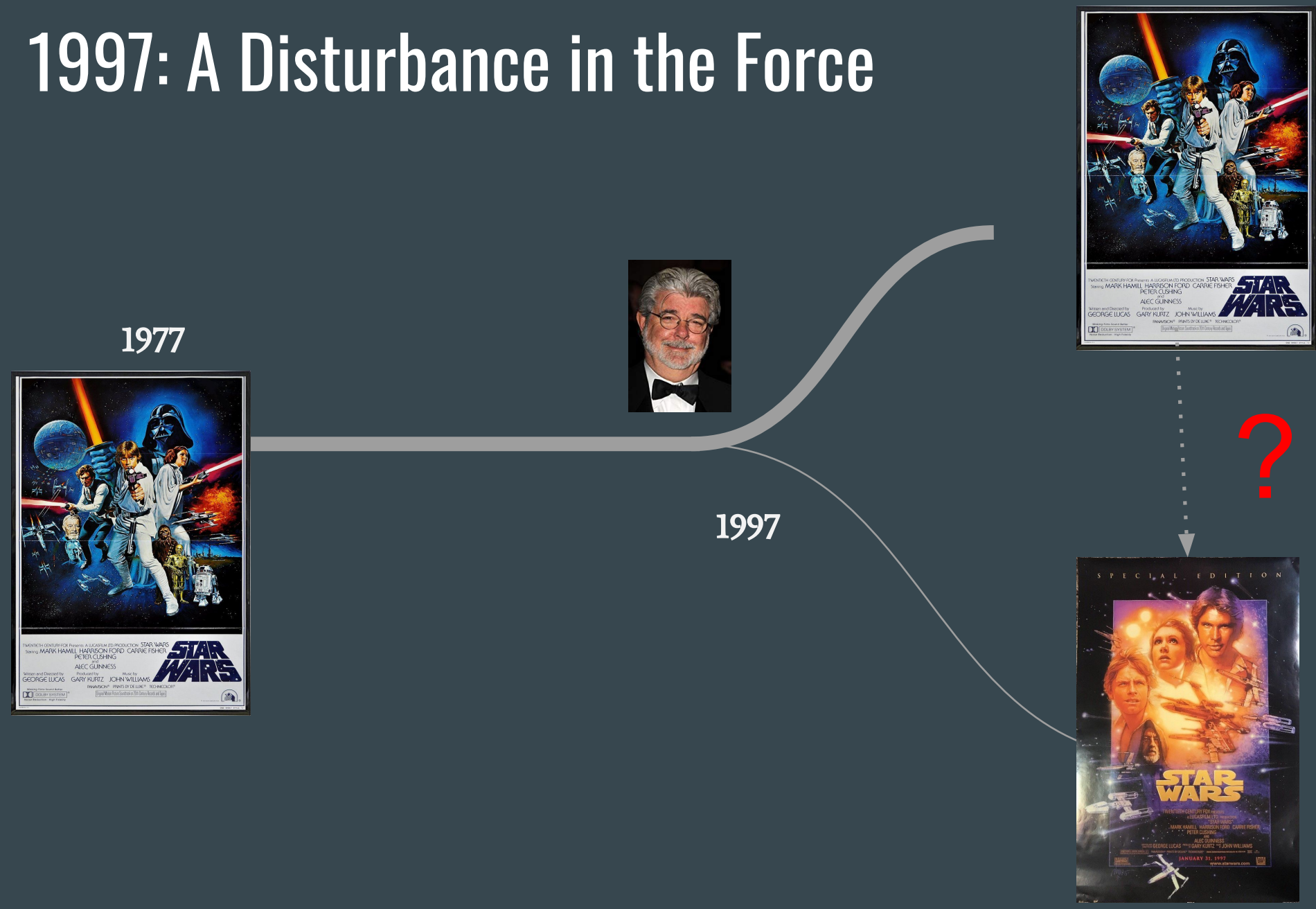


\section{What does this mean for Data?}

Philosophically, no issue with

versions of a creative work.

dcterms:isVersionOf

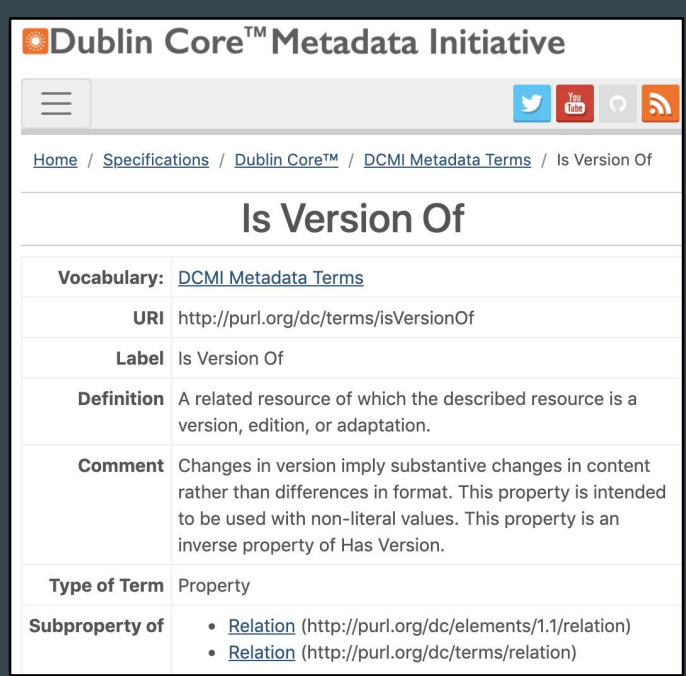

How do we explain what happened to those who don't know yet? 


\section{A Case of 'st1_50m'}

An observation made at the location of

station ' 1 ' at a depth of 50 meters

\begin{tabular}{|c|c|c|c|c|c|c|c|c|c|c|c|c|}
\hline Dest_hit_annotation & best_hit_taxon_id & st1 $050 \mathrm{~m}$ & st1_090m & st1_120m & st1_200m & st1_300m & st1_400m & st1_600m & st3_040m & st3_060m & st3_120m & st3 \\
\hline & 247490 & 0 & 0 & 0 & 0 & 122 & 121 & 116 & 0 & 0 & 17 & \\
\hline ctase subunit beta (E & 330214 & 0 & 0 & 0 & 1 & 136 & 173 & 153 & 0 & 0 & 18 & \\
\hline .9); K04077 chapero & 167546 & 80 & 91 & 59 & 35 & 2 & 2 & 1 & 60 & 44 & 24 & \\
\hline CsoS1 & 167555 & 155 & 162 & 94 & 38 & 0 & 0 & 0 & 54 & 40 & 39 & \\
\hline bstrate binding prot & 167546 & 202 & 203 & 169 & 158 & 26 & 30 & 19 & 100 & 86 & 27 & \\
\hline nily & 859653 & 7 & 7 & 8 & 7 & 51 & 69 & 74 & 3 & 1 & 19 & \\
\hline & 314261 & 17 & 20 & 19 & 9 & 30 & 35 & 36 & 12 & 15 & 22 & \\
\hline g protein; family 5 & 89187 & 0 & 0 & 0 & 0 & 50 & 50 & 65 & 0 & 0 & 2 & \\
\hline mate--ammonia liga: & 146891 & 62 & 60 & 54 & 58 & 3 & 4 & 2 & 60 & 53 & 4 & \\
\hline rate-binding protein & 913324 & 3 & 2 & 2 & 4 & 33 & 78 & 43 & 0 & 0 & 6 & \\
\hline & 93058 & 57 & 63 & 76 & 34 & 5 & 4 & 3 & 39 & 40 & 47 & \\
\hline mic substrate-bindin & 375451 & 0 & 2 & 3 & 0 & 41 & 48 & 44 & 0 & 0 & 6 & \\
\hline sparagine $\mathrm{ABC}$ trans & 488538 & 2 & 7 & 11 & 2 & 31 & 52 & 44 & 2 & 3 & 5 & \\
\hline & 1090946 & 1 & 1 & 7 & 0 & 51 & 47 & 37 & 0 & 0 & 5 & \\
\hline & 859653 & 88 & 68 & 33 & 29 & 1 & 4 & 3 & 38 & 32 & 10 & \\
\hline oxylase; K01601 ribı & 146891 & 46 & 57 & 40 & 36 & 1 & 3 & 0 & 37 & 41 & 15 & \\
\hline & 1073573 & 20 & 10 & 2 & 1 & 26 & 44 & 29 & 10 & 8 & 2 & \\
\hline Ipha (EC:1.2.1.2); KO & 639282 & 0 & 0 & 0 & 0 & 37 & 41 & 36 & 0 & 0 & 3 & \\
\hline rate-binding protein. & 644966 & 0 & 0 & 0 & 0 & 29 & 38 & 31 & 0 & 0 & 1 & \\
\hline nily & 859653 & 25 & 16 & 15 & 9 & 50 & 55 & 41 & 12 & 14 & 19 & \\
\hline sparagine $\mathrm{ABC}$ trans & 488538 & 0 & 14 & 18 & 0 & 36 & 45 & 33 & 0 & 0 & 22 & \\
\hline & 1073573 & 4 & 5 & 1 & 2 & 35 & 27 & 35 & 0 & 0 & 3 & \\
\hline
\end{tabular}




\section{Putting the 'F-I-R' in FAIR}

Findable

- get all datasets that recorded 'station' or 'depth'

Interoperable

- linked to community vocabs

Reusable

- metadata with provenance

How 'st1_50m' and all 'st(\#)_(\#)m' became 'station' and 'depth'

\begin{tabular}{|r|r|r|}
\hline station & depth & spectral_count \\
\hline 8 & 200 & 0 \\
\hline 9 & 40 & 12 \\
\hline 9 & 70 & 4 \\
\hline 9 & 380 & 0 \\
\hline 12 & 40 & 0 \\
\hline 12 & 120 & 0 \\
\hline 12 & 300 & 0 \\
\hline 1 & 50 & 0 \\
\hline 1 & 90 & 0 \\
\hline 1 & 120 & 0 \\
\hline 1 & 200 & 0 \\
\hline 1 & 300 & 0 \\
\hline 1 & 400 & 0 \\
\hline 1 & 600 & 0 \\
\hline 3 & 40 & 9 \\
\hline 2 & 60 & 0 \\
\hline
\end{tabular}




\section{A Tale of Two Versions}

\section{'st(\#)_(i)m'}

original

data

original

Data

\section{'station','depth'}

updated

Data 


\section{A Tale of Two Versions}

\section{'st( $(i) \_(\#) m^{\prime}$}

original

data

\section{original \\ Data}

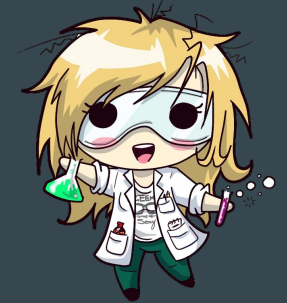

\section{'station','depth'}

updated

Data 


\section{How do we explain what happened to those who don't know?}

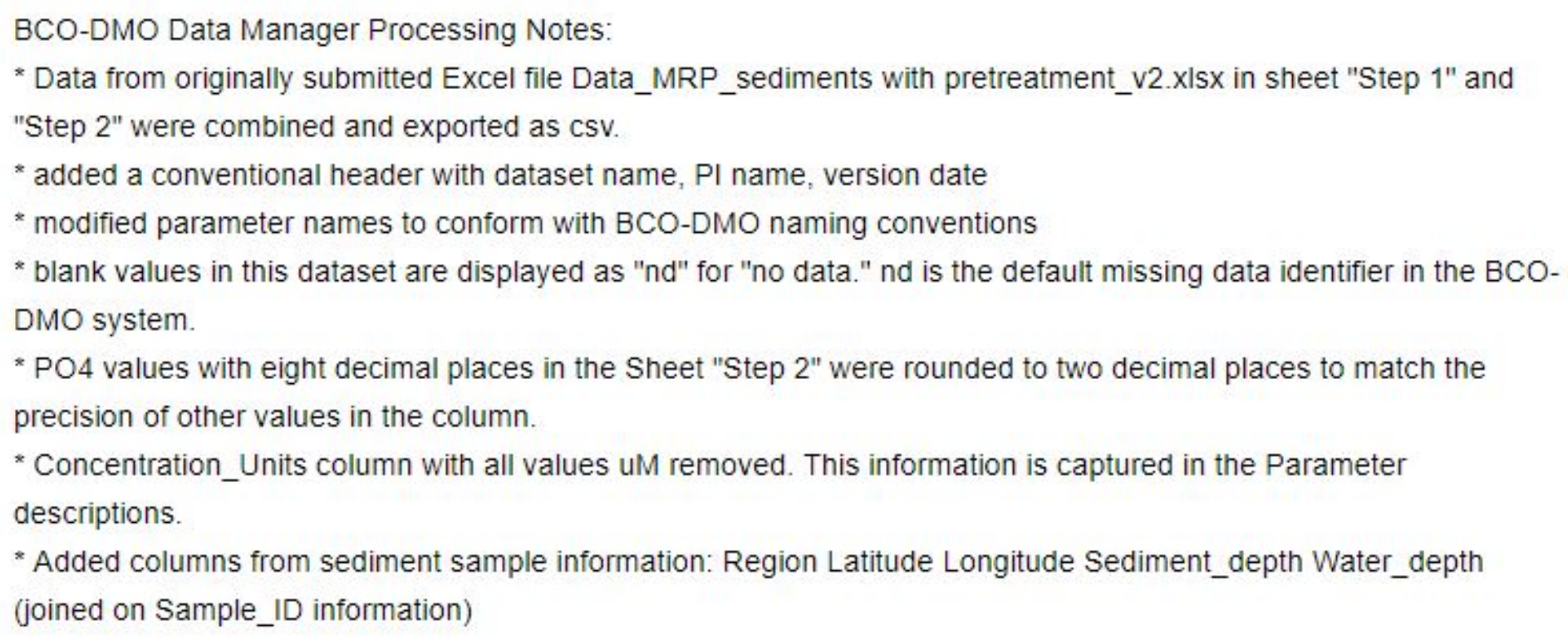




\section{How do we explain what happened}

to those who don't know?

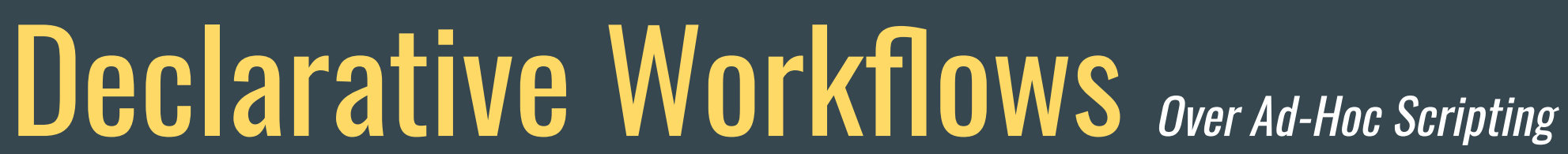




\section{Declarative Workflows - A set of steps to execute}

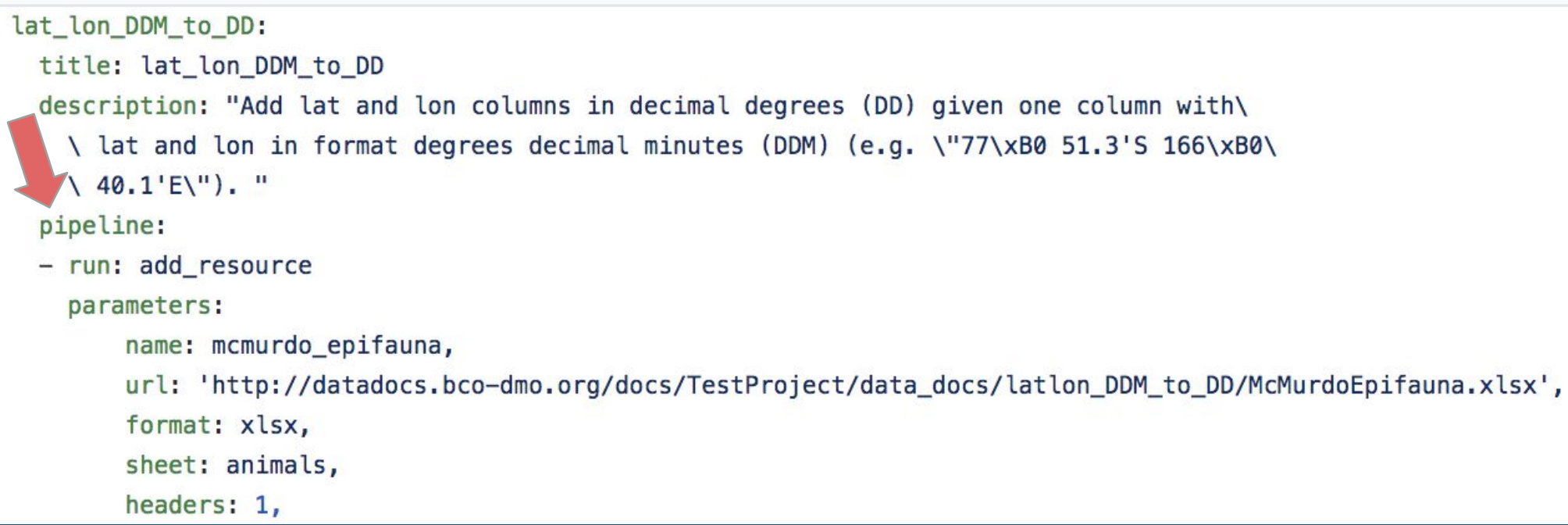




\section{Declarative Workflows - Each step is "named"}

lat_lon_DDM_to_DD:

title: lat_lon_DDM_to_DD

description: "Add lat and lon columns in decimal degrees (DD) given one column with\

$\backslash$ lat and lon in format degrees decimal minutes (DDM) (e.g. \"77\xB0 51.3'S 166\xB0

\40.1'E\")."

pipeline:

- run: add_resource parameters:

name: mcmurdo_epifauna,

url: 'http://datadocs.bco-dmo.org/docs/TestProject/data_docs/latlon_DDM_to_DD/McMurdoEpifauna.xlsx' , format: $x l s x$,

sheet: animals,

headers: 1 , 


\section{Declarative Workflows - Each step has inputs}

lat_lon_DDM_to_DD:

title: lat_lon_DDM_to_DD

description: "Add lat and lon columns in decimal degrees (DD) given one column with\

$\backslash$ lat and lon in format degrees decimal minutes (DDM) (e.g. \"77\xB0 51.3'S 166\xB0

\40.1'E\")."

pipeline:

- run: add_resource parameters:

name: mcmurdo_epifauna,

url: 'http://datadocs.bco-dmo.org/docs/TestProject/data_docs/latlon_DDM_to_DD/McMurdoEpifauna.xlsx' , format: $x$ lsx,

sheet: animals,

headers: 1 , 


\section{Declarative Workflows - More steps}

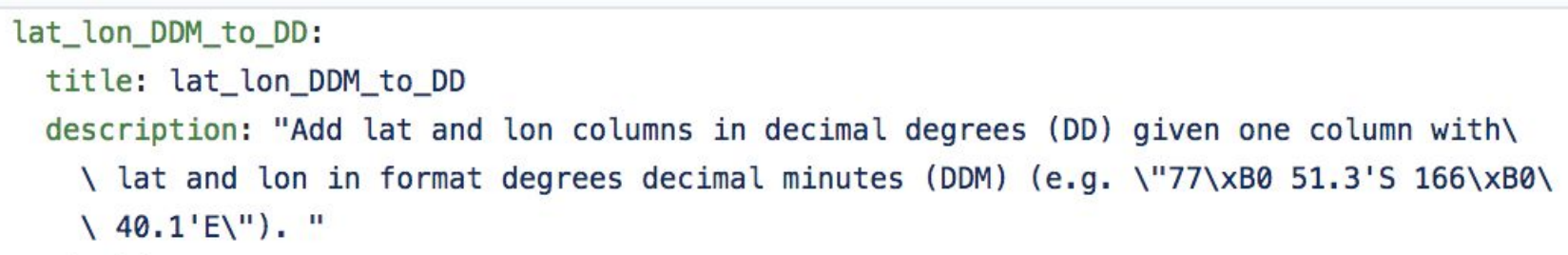

- run: bcodmo_pipeline_processors.convert_to_decimal_degrees cache: True parameters:

resources: [mcmurdo_epifauna]

fields:

- - input_field: lat_long, format: degrees-decimal_minutes, output_field: lat_converted, directional: '',

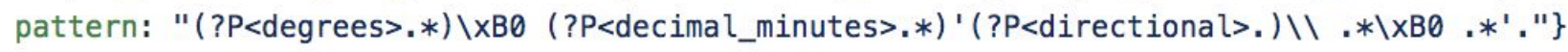

- run: bcodmo_pipeline_processors.convert_to_decimal_degrees

cache: true

parameters:

resources: [mcmurdo_epifauna]

fields:

- \{input_field: lat_long, format: degrees-decimal_minutes, output_field: long_converted, directional: '',

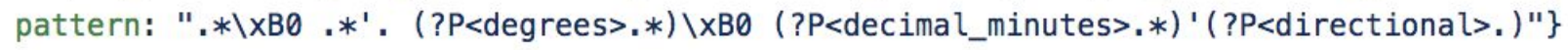




\section{Declarative Workflows - Names identify code to execute}

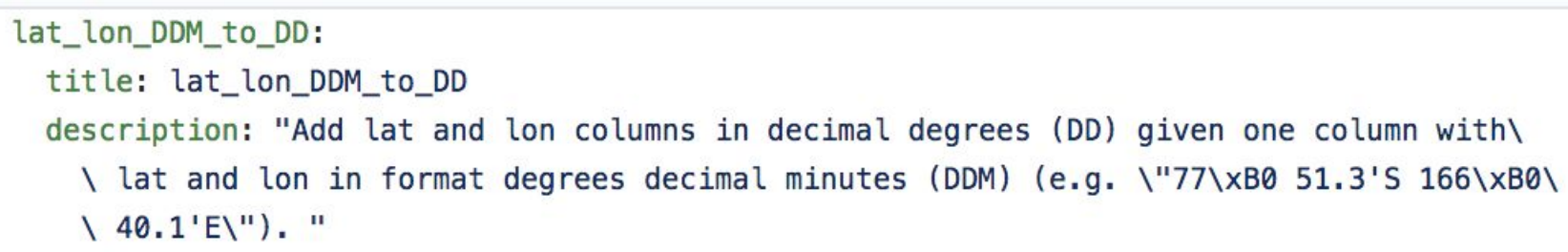

- run: bcodmo_pipeline_processors.convert_to_decimal_degrees cache: True parameters:

resources: [mcmurdo_epifauna]

fields:

- \{input_field: lat_long, format: degrees-decimal_minutes, output_field: lat_converted, directional: ' ',

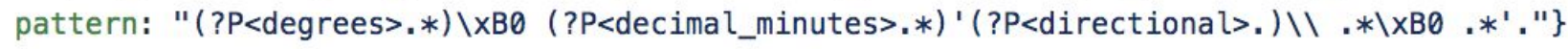

- run: bcodmo_pipeline_processors.convert_to_decimal_degrees cache: true

parameters:

resources: [mcmurdo_epifauna]

fields:

- \{input_field: lat_long, format: degrees-decimal_minutes, output_field: long_converted, directional: '',

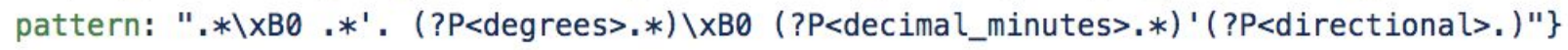




\section{Declarative Workflows - Each step has its own inputs}

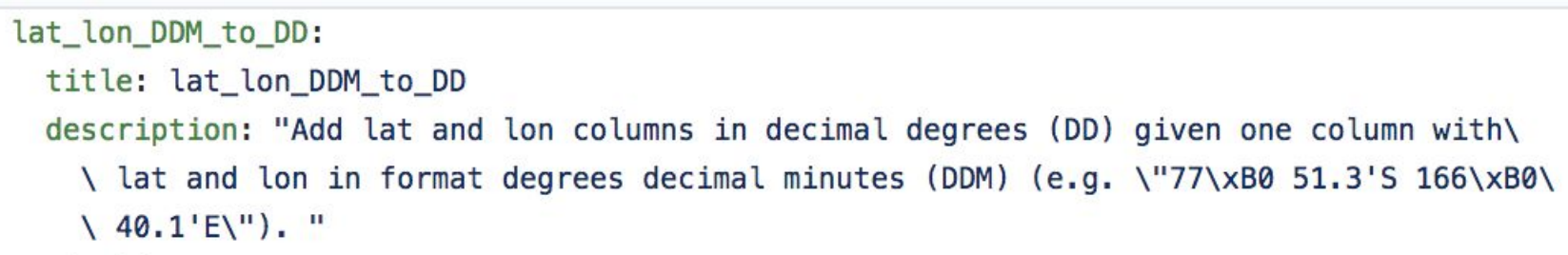

- run: bcodmo_pipeline_processors.convert_to_decimal_degrees cache: True parameters:

resources: [mcmurdo_epifauna]

fields:

- \{input_field: lat_long, format: degrees-decimal_minutes, output_field: lat_converted, directional: ' ',

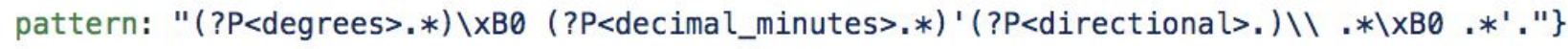

- run: bcodmo_pipeline_processors.convert_to_decimal_degrees cache: true parameters:

resources: [mcmurdo_epifauna]

fields:

- \{input_field: lat_long, format: degrees-decimal_minutes, output_field: long_converted, directional: '',

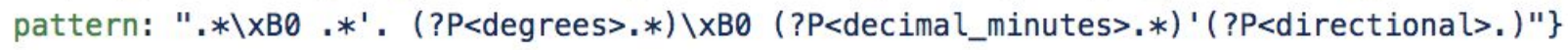




\section{Example: Convert latitude format}

- run: bcodmo_pipeline_processors.convert_to_decimal_degrees cache: True

parameters:

resources: [mcmurdo_epifauna]

fields:

- \{input_field: lat_long, format: degrees-decimal_minutes, output_field: lat_converted, directional: ' ',

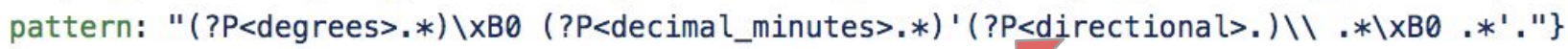

linput_field: lat_long, format: degrees-decimal_minutes, output_field: lat_converted, direction

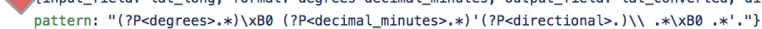
- run: bcodno pipeline processors, convert to_decinat degrees cache: true
parameters:

parameters:
resources:

fields:

murdo_epifauna]

- \{input_field: lat_long, format: degrees-decimal_minutes, output_field: long_converted, directiona

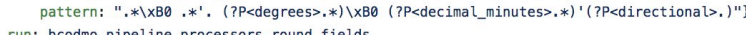

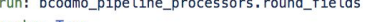

cache: True
parameters:

resources: [mcmurdo_epifauna] 


\section{Example: Convert latitude format}

- run: bcodmo_pipeline_processors.convert_to_decimal_degrees cache: True

parameters:

resources: [mcmurdo_epifauna]

fields:

- \{input_field: lat_long, format: degrees-decimal_minutes, output_field: lat_converted, directional: ' ',

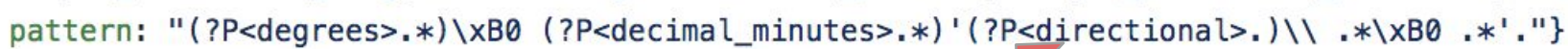

\section{lat_long}

$77^{\circ} 51.1^{\prime} \mathrm{S} 166^{\circ} 40^{\prime} \mathrm{E}$

$77^{\circ} 51^{\prime} \mathrm{S} 166^{\circ} 39.7^{\prime} \mathrm{E}$

$77^{\circ} 51^{\prime} \mathrm{S} 166^{\circ} 39.7^{\prime} \mathrm{E}$

$77^{\circ} 51^{\prime} \mathrm{S} 166^{\circ} 39.7^{\prime} \mathrm{E}$

$77^{\circ} 51^{\prime} \mathrm{S} 166^{\circ} 39.6^{\prime} \mathrm{E}$

$77^{\circ} 51^{\prime} \mathrm{S} 166^{\circ} 39.6^{\prime} \mathrm{E}$

$77^{\circ} 51^{\prime} \mathrm{S} 166^{\circ} 39.6^{\prime} \mathrm{E}$

$77^{\circ} 50.9^{\prime} \mathrm{S} 166^{\circ} 39.4^{\prime} \mathrm{E}$ lat_dd lon_dd

$-77.855166 .6683$

$-77.855166 .6683$

$-77.855166 .6683$

$-77.8517 \quad 166.6667$

\begin{tabular}{l|l}
-77.8517 & 166.6667
\end{tabular}

\begin{tabular}{l|l}
-77.8517 & 166.6667
\end{tabular}

$-77.85166 .6617$

$-77.85166 .6617$ cache: True

resources: [memurdo_epifauna]

missingvatues: ["nd"]

un: bcodmo_pipeline_processors. convert_to_decimal_degrees cache: True

resources: [mcmurdo_epifauna)

input_field: lat_long, format: degrees-decimal_minutes, output_field: lat_converted, directional

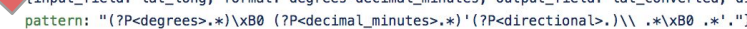
run: bcodmo pipeline processors, coovert_to decint_degres cache: true

parameters:

resources: [mcmurdo_epifauna]

fields:

- \{input_field: lat_long, format: degrees-decimal_minutes, output_field: long_converted, directiona

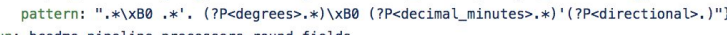
un: beodno_pipeline_processors.round_tlelds

cache: True

resources: [mcmurdo_epifaunal

fields:

\{digits: 5, name: lat_convert

fun: bcodmo_pipeline_processors. round_fields 


\section{Declarative Workflows: Focus on 'What' to do}

- Describe the steps of transformation ('what' to do)

- Software/code interpret these steps ('how' best to do it)

\begin{tabular}{|l|l|}
\hline Formats of 'What' (Data) & Formats of 'How' (Code) \\
\hline JSON & Python \\
YAML & Java \\
$\begin{array}{l}\text { Sometimes even natural language } \\
\text { etc... }\end{array}$ & Javascript \\
& etc... \\
\hline
\end{tabular}

The 'What' abstracts the implementation of 'How'. 


\section{Why not just (re)use code?}

$\rightarrow$ Easier to teach non-coders

- 'what' should be done over 'how' to do it

$\rightarrow$ As data,

$\checkmark$ longer shelf-life than code

- code can be changed/swapped without impacting DM processes

$\checkmark$ easier to manage (query \& assess)

Q: All datasets that used: 'convert_to_decimal_degress'

Declarative workflows can serve as provenance. 


\section{Workflow as PROV}

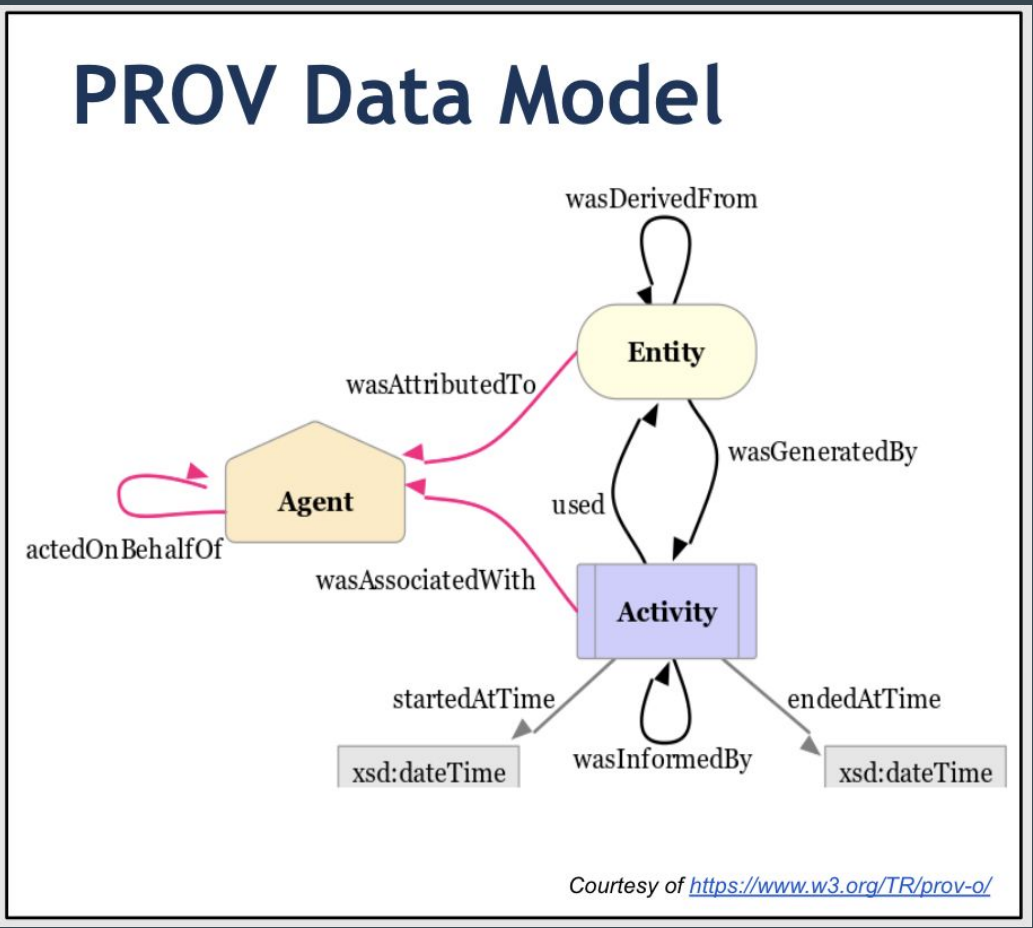

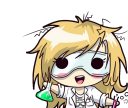

Data Manager

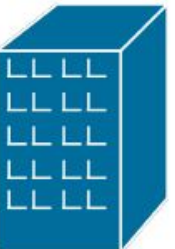

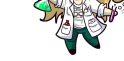

actedOnBehalfof
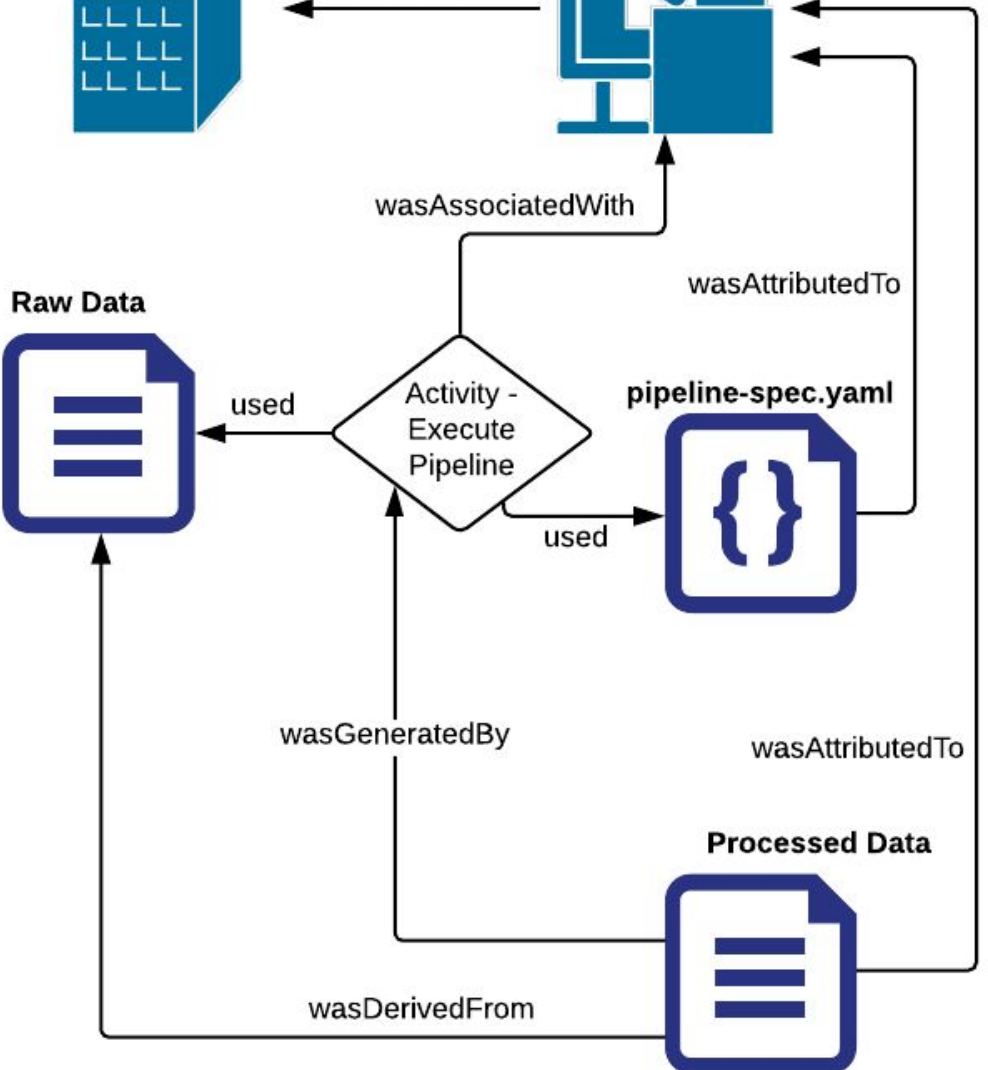


\section{What use is this PROV?}

The provenance is data describing how the archived version changed the original

original

data

original

data

archived

data 


\section{What use is this PROV?}

The provenance is data describing how the archived version changed the original

original

data

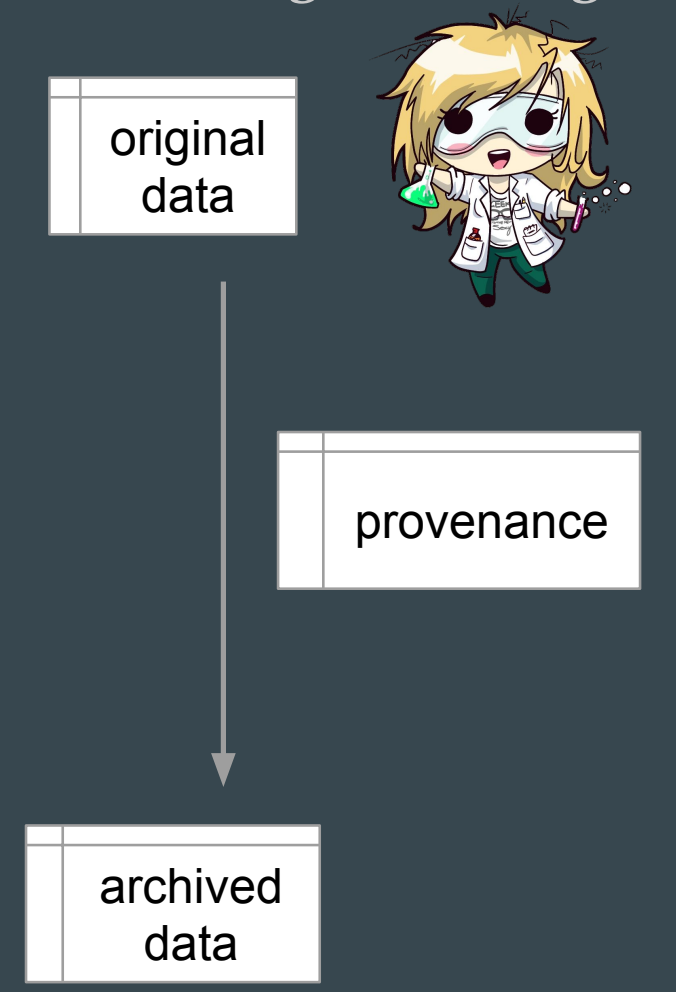




\section{What use is this PROV?}

The provenance is data describing how the archived version changed the original

original

data

\section{FAIR}

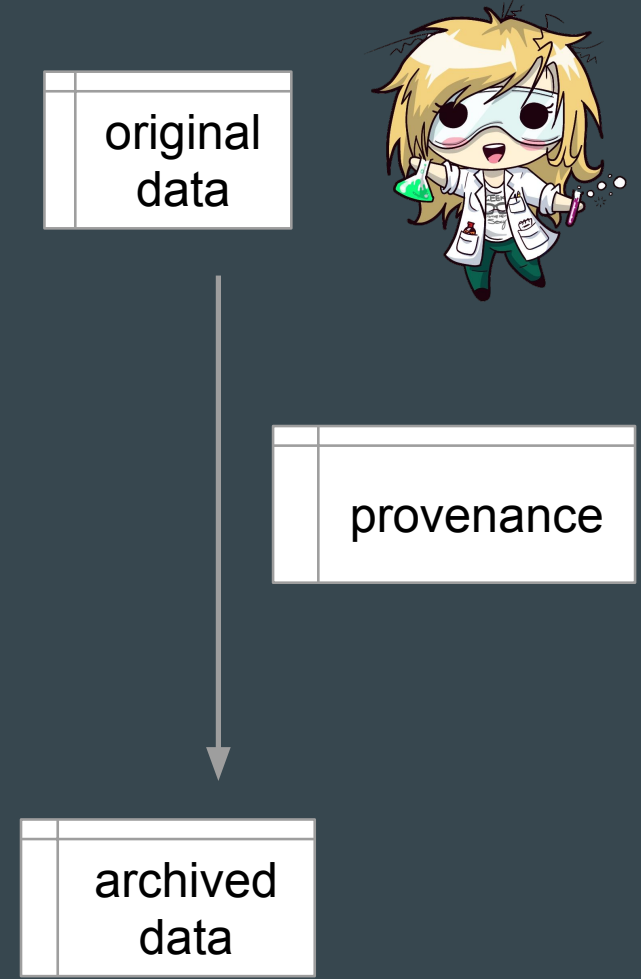




\section{Workflow Tools at BCO-DMO}

frictionlessdata.io

pypi.org/project/dataflows

github.com/BCODMO/bcodmo_processors

frictionlessdata.io/blog/2020/02/10/frictionless-data-pipelines-for-open-ocean/ 


\section{Laminar Demo}




\section{QUESTIONS?}

FRICTIONLESS DATA

SPECIFICATIONS AND SOFTWARE

frictionlessdata.io pypi.org/project/dataflows github.com/BCODMO/bcodmo_processors

frictionlessdata.io/blog/2020/02/10/frictionless-data-pipelines-for-open-ocean/ 